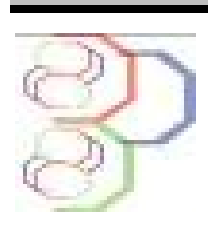

Journal of Applied Biosciences 104:9947 - 9954

ISSN 1997-5902

\title{
Les attaques des Termites (Isoptera) dans les parcelles de reboisement de la Grande Muraille Verte au Sénégal
}

\author{
SAMB Tamsire ${ }^{1}$, CISSÉ Ababacar², NDIAYE Abdoulaye Baïla ${ }^{3}$ \\ ${ }^{1}$ Institut fondamental d'Afrique noire Cheikh Anta Diop, laboratoire de zoologie des invertébrés terrestres, BP 206 Dakar- \\ Fann, Sénégal, \\ 2Institut fondamental d'Afrique noire Cheikh Anta Diop, laboratoire de botanique, \\ 3Institut fondamental d'Afrique noire Cheikh Anta Diop, laboratoire de zoologie des invertébrés terrestres. \\ Correspondant auteur E-mail : tam2samb@hotmail.com
}

Original submitted in on 23rd April 2016. Published online at www.m.elewa.org on 31st August 2016

http://dx.doi.org/10.4314/jab.v104i1.7

\section{RÉSUMÉ}

Objectif. Pour une meilleur compréhension des interactions entre Termites et ligneux dans les parcelles de reboisement de la Grande Muraille Verts au Sénégal, nous nous sommes intéressé à l'attaques des Termites sur les ligneux.

Méthodologie et résultats. Dans chaque parcelle, une superficie d'un hectare a été délimitée. Le nombre d'espèces ligneuses, ainsi que le nombre d'individus de chaque essence attaqués ont été dénombrés. Les espèces de Termites qui les attaquent ont été inventoriées. Cette étude a permis de recenser 15 espèces ligneuses attaquées par 14 espèces de Termites. Les espèces les plus attaquées sont Boscia senegalensis et Balanites aegyptiaca. Les espèces du genre Acacia ne sont attaquées que sur les parties dont l'écorce a été enlevée. Les espèces des genres Psammotermes, Microcerotermes et Odontotermes s'attaquent plus aux ligneux.

Conclusion et application des résultats : Cette étude a permis de connaitre d'avantage la diversité des termites et des essences végétales dans les parcelles de reboisement mais aussi les interactions qui existent entre les termites est les essences végétales.

Mots Clés : Termites, ligneux, attaques, zone semi-aride

\section{ABSTRACT}

Objective: To better understand interactions between Termites and ligneous in the plots of reafforestation in the Great Green Wall of Senegal, we are interested in Termites attacks on ligneous.

Methodology and results: In each plot a surface of one hectare was delimited. The number of ligneous species as weel as the number of individual of each attacked spicy were counted. The termites species that attack them were made an inventory of this survey has allowed to make an inventory of fifteen ligneous species attacked by fourteen Termites species. The most attacked species are Boscia senegalensis and Balanites aegyptiaca. The species like Acacia are only attacked on the sides the bark of which was abstracted. The species like (such as) Psammotermes, Microcerotermes and Odontotermes attack most on ligneous. 


\section{Samb et al. J. Appl. Biosci. 2016 Les attaques des Termites (Isoptera) dans les parcelles de reboisement}

de la Grande Muraille Verte au Sénégal

Conclusion and Application of results : The study to know the advantage of diversity of termites and plant species in reforestation plots but also the interactions between termites is the plant species

Key words termites, ligneous, attacks, half dry area.

\section{INTRODUCTION}

En zone sahélo-saharienne, le déficit pluviométrique et l'action anthropique ont fortement perturbé le fonctionnement des écosystèmes entrainant une perte importante de la biodiversité et de ressources naturelles essentielles à la survie des populations autochtones. Pour inverser cette tendance, Union africaine a adopté lors de son 8ème sommet en janvier 2007 l'initiative de la Grande Muraille Verte $d u$ Sahara dont l'objectif est de freiner l'avancée du désert (www.grandemurailleverte.org 6 avril 2011) La Grande Muraille Verte a pour ambition de redonner aux écosystèmes sahélo-sahariens une structure et une fonctionnalité permettant la production de ressources naturelles diverses et suffisantes pour satisfaction durable des besoins des populations. Au Sénégal, les activités de reboisement et les aménagements d'infrastructures dans la région ciblée s'accompagnent nécessairement de perturbations du milieu. Selon Wood 1975, les Termites, groupe le plus important de la macrofaune des sols tropicaux constituent un des modèles les plus intéressants pour la compréhension des changements attendus dans le milieu. Le comportement de cette macrofaune est très varié selon le groupe trophique et induit d'importantes modifications à la pédogenèse et la couverture végétale (Samb \& al., 2014). Le système complexe de leur prise de nourriture, la construction de leur nid épigé, partiellement ou entièrement

\section{MÉTHODOLOGIE}

Zone d'étude : L'étude est réalisée dans le Ferlo Nord du Sénégal, dans les parcelles de reboisement de la Grande Muraille Verte situées entre Widou Thiengoly et Téssékéré (figure 1). C'est une zone semi-aride avec une savane sahélienne. Cette partie Sénégalais est présentée comme étant la plus aride du pays (Ndiaye \& Samb, 2012). Dans cette région règne un climat régional sahélien intermédiaire entre les climats saharien et soudanien. En fonction de la pluviométrie, facteur le plus important dans l'individualisation des domaines climatiques, le domaine sahélien est délimité par les hypogé et la nature diversifiée de leur alimentation sont les principaux facteurs qui influencent la décomposition de la matière organique du sol et la structure du sol (Samb \& al., 2014). En 2012, Okullo \& Moe ont montré que dans une savane du Sudouest de l'Ouganda, l'effet des Termites sur la strate herbacée est beaucoup plus important que celui des grands herbivores pourtant dominant en termes de biomasse. La nuisance des Termites aux arbres fruitiers, aux bois ouvrés, aux bibliothèques, aux denrées alimentaires. Est pourtant réelle. Ce qui leur a valu le qualificatif de " plaie des pays chauds " par Grassé (1949). Les premiers travaux sur les Termites dans le Ferlo Nord ont été réalisés par Lepage (1974) à Fété Olé au Nord-ouest de Widou Thingoly. Plus récemment, Ndiaye \& Samb (2012), Guèye (2011) et Samb et al. (2010) ont étudié la faune de Termites respectivement à Widou-Téssékéré (Parcelles de reboisement), Katané (Réserve de Faune du Ferlo Nord) et à Danthiady (Matam). Pour une meilleure compréhension du fonctionnement des écosystèmes dans les parcelles de reboisement de la Grande Muraille Vert au Sénégal, nous nous intéressons aux interactions pouvant exister entre Termites et espèces végétales. Dans ce travail nous avons procédé à la caractérisation des espèces de Termites et des espèces végétales qui sont attaquées par ces Termites.

isohyètes $250-300 \mathrm{~mm}$ et $500-550 \mathrm{~mm}$. Cette tranche d'eau est précipitée en une seule saison, puisque le Heug, qui correspond peut-être à la deuxième saison des pluies des pays équatoriaux, est ici très peu sensible, ou même inexistant. La végétation du domaine sahélien, qui fait la transition entre les végétations saharienne et soudanienne, est caractérisée par des espèces ligneuses peu nombreuses, souvent rabougries, la plupart épineuses, à rameaux souvent étalés en parasol, à feuillage souvent réduit et caduc (Michel et al., 1969). 

de la Grande Muraille Verte au Sénégal

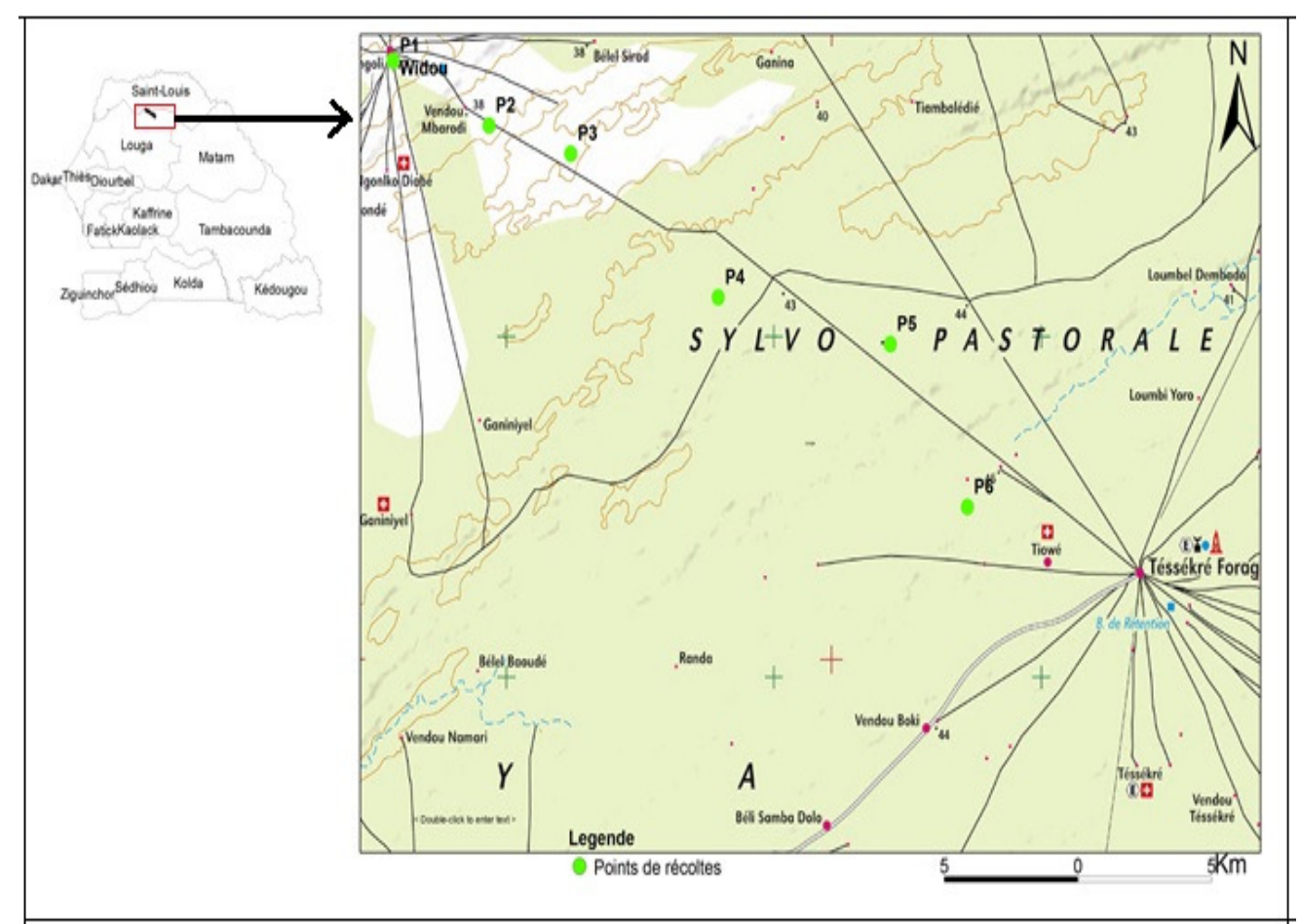

Figure1 : Localisation de la zone d'étude

Méthode d'étude : L'étude a été réalisée en mars et en juin 2015. Nous avions travaillé dans six (06) parcelles reboisées et clôturées situées entre Widou Thiengoly et Téssékéré. Dans chaque parcelle, une superficie d'un hectare a été délimitée. Au niveau de chaque hectare délimité, on commence d'abord à relever les coordonnées géographiques. Ensuite on compte le nombre de pieds d'espèces végétales ligneuses attaquées. Enfin, pour chacune des espèces végétales attaquées, on cherche l'espèce de Termite qui l'attaque et la partie de la plante qui est attaquée (racines, tige, branches ou feuilles). Pour les essences végétales attaquées, les Termites sont cherchés sur les racines, sur les tiges, sous les écorces, sur les branches vivantes ou mortes. Les Termites sont récoltés dans des tubes contenant de l'éthanol $95^{\circ}$ avec une étiquette portant une référence (Lieu, date, parcelles, et 1 'endroit de récolte). L'identification des spécimens de Termites a été faite au laboratoire de zoologie des invertébrés terrestres de I'Institut Fondamental d'Afrique Noire Ch. A. Diop (IFANCh. A. Diop) de Dakar par comparaison avec des spécimens de référence sur les Termites et également avec des ouvrages, de Silvestri (1914-15), de Sjöstedt (1925) et de Bouillon \& Mathot (1965). La liste des
Termites identifiés est présentée selon la classification d'Inward et al. (2007). L'identification des espèces végétales a été faite le plus souvent sur place à l'aide de la Flore du Sénégal (Berhaut, 1967), d'autres ouvrages de références (Adam, 1970 ; Baumer, 1995 ; Arbonnier, 2000). Des échantillons sont prélevés une comparaison avec des échantillons d'herbier de la collection de l'IFAN. Fréquence : La fréquence des espèces est un facteur quantitatif qui exprime le nombre de pieds attaqués sur le nombre total de pieds ou encore le nombre de relevés où l'espèce est rencontrée sur le nombre total de relevés. Elle est obtenue par l'application de la formule suivante.

$$
\mathrm{F}=\frac{N i}{N t} \times 100
$$

$\mathrm{Ni}$ le nombre de relevés contenant l'espèce et $\mathrm{Nt}$ le nombre total de relevés échantillonnés.

Analyse statistique des données: L'analyse de la variance (ANOVA) et la comparaison des moyennes (Newman-Keuls) ont été effectuées sur les données recueillies avec le logiciel XLSTAT 6.1.9 au seuil de 5\% pour évaluer le degré de signification des différences observées. 


\section{Samb et al. J. Appl. Biosci. 2016 Les attaques des Termites (Isoptera) dans les parcelles de reboisement de la Grande Muraille Verte au Sénégal}

\section{RÉSULTATS}

Espèces de Termites rencontrées: La liste et la position systématique des espèces inventoriées de Termites dans la zone d'études sont représentées dans

le tableau 1. L'étude qualitative a permis de recenser 14 espèces de termites réparties dans 9 genres, 4 sousfamilles et 2 familles.

Tableau 1 : Espèces de termites récoltées et position systématique des espèces

\begin{tabular}{|c|c|c|}
\hline Famille & Sous-famille & Espèce \\
\hline \multirow[t]{2}{*}{ Rhinotermitidae Light, 1912} & Coptotermitinae Holmgren, 1911 & Coptotermes intermedius Silvestri, 1912 \\
\hline & Psammotermitinae Desneux, 1902 & Psammotermes hybostoma Desneux, 1902 \\
\hline \multirow[t]{12}{*}{ Termitidae Westwood, 1840} & \multirow[t]{6}{*}{ Termitinae Sjöstedt, 1926} & Amitermes evuncifer Silvestri, 1912 \\
\hline & & Amitermes messinae Fuller 1922 \\
\hline & & Angulitermes nilensis Harris, 1962 \\
\hline & & Microcerotermes spl \\
\hline & & Microcerotermes spll \\
\hline & & Promirotermes holmgreni infera Silvestri, 1914-1915 \\
\hline & \multirow[t]{6}{*}{ Macrotermitinae Kemner, 1934} & Macrotermes subhyalinus Rambur, 1842 \\
\hline & & Microtermes grassei Ghidini, 1955 \\
\hline & & Microtermes lepidus Sjöstedt, 1924 \\
\hline & & Microtermes subhyalinus Silvestri, 1914 \\
\hline & & Odontotermes spl \\
\hline & & Odontotermes spll \\
\hline
\end{tabular}

La richesse générique semble importante alors que la richesse spécifique est moyenne. Toutes les espèces recensées par cette étude ont été signalées antérieurement dans la zone. Pour ce qui est des genres Microcerotermes et Odontotermes, l'état de confusion qui règne dans leur systématique ne permet pas, à l'heure actuelle, une discrimination objective des espèces citées dans la littérature. La révision de ces genres est nécessaire. En fonction du régime trophique, les espèces de Termites identifiées se répartissent entre lignivores, champignonnistes et humivores. Les lignivores $(42,86 \%)$ appartiennent aux genres Coptotermes, Psammotermes, Amitermes et Microcerotermes. Les humivores (14,28\%) sont représentées par Angulitermes nilensis et Promirotermes holmgreni. Les champignonnistes $(42,86 \%)$ sont représentées par les genres Macrotermes, Microtermes et Odontotermes.

Tableau 2 : Espèces végétales attaquées par les Termites dans les biotopes étudiés

\begin{tabular}{|l|l|}
\hline Familles & Espèces \\
\hline Anacardiaceae & Sclerocarya birrea (A.Rich.) Hochst \\
\hline Apocynaceae & Calotropis procera (Aiton) W.T.Aiton \\
\hline Asclepiadaceae & Leptadagna pirotechnica (Pers.) Decne. \\
\hline Balanitaceae & Balanites aegyptiaca (L.) Delile \\
\hline Bombacaceae & Adansonia digitata L. \\
\hline Cappraceae & Boscia senegalensis (Pers.) Lam. \\
\hline Combretaceae & Anogeissus leiocarpa (DC.) Guill. \& Perr. \\
\cline { 2 - 2 } & Combretum glutinosum Perr.ex DC. \\
\cline { 2 - 2 } & Guiera senegalensis J.F.Gmel \\
\hline \multirow{3}{*}{ Mimosaceae } & Acacia raddiana Savi / tortilis (Forsk.) Hayne \\
\cline { 2 - 2 } & Acacia senegal L. (Willd.) \\
\cline { 2 - 2 } & Acacia seyal Delile \\
\cline { 2 - 2 } & Faidherbia albida (Delile) A. Chev. \\
\hline Rubiaceae & Mitragyna inermis (Willd.) K. Schum. \\
\hline Tiliaceae & Grewia bicolor Juss. \\
\hline
\end{tabular}


Espèces ligneuses attaquées : La liste des espèces végétales attaquées par les Termites dans les différentes parcelles étudiées est représentée par le tableau 2. Un total de 15 espèces végétales appartenant à 10 familles a été listé. Les Mimosaceae sont les plus représentés avec $26,66 \%$ suivis des Combretaceae avec $20 \%$. Les autres familles ont chacune un pourcentage de $6,66 \%$.

Fréquences des espèces de Termites dans les différentes parcelles (Tableau 3) : L'analyse descriptive des données obtenues sur la récolte des Termites a permis de constater que ce sont les genres
Psammotermes, Microcerotermes et Odontotermes qui s'attaquent les plus aux ligneux avec des fréquences respectives de $21,36 \%, 20,39 \%$ et $20,1 \%$. La fréquence des attaques des genres Microtermes et Amitermes sont $10,67 \%$ et $8,74 \%$. L'espèce Macrotermes subhylinus a une fréquence d'attaque de $3,88 \%$. Les espèces Angulitermes nilensis et Coptotermes intermedius ont chacune une fréquence d'attaque de 1,94\%. L'espèce Promirotermes holmgreni est celle qui attaque le moins avec une fréquence de $0,97 \%$.

Tableau 3 : Fréquence des espèces de Termites

\begin{tabular}{l|l|l|l}
\hline Modalité & Effectif & $\%$ & Fréquence. réelle. \\
\hline Amitermes evuncifer & 7 & 6,80 & 0,068 \\
Amitermes messinae & 2 & 1,94 & 0,019 \\
Angulitermes nilensis & 2 & 1,94 & 0,019 \\
Coptotermes intermedius & 2 & 1,94 & 0,019 \\
Macrotermes subhyalinus & 4 & 3,88 & 0,039 \\
Microcerotermes spl & 9 & 8,74 & 0,087 \\
Microcerotermes spll & 12 & 11,65 & 0,117 \\
Microtermes grassei & 5 & 4,85 & 0,049 \\
Microtermes lepidus & 3 & 2,91 & 0,029 \\
Microtermes subhyalinus & 3 & 2,91 & 0,029 \\
Odontotermes spl & 18 & 17,48 & 0,175 \\
Odontotermes spll & 13 & 12,62 & 0,126 \\
Promirotermes holmgreni & 1 & 0,97 & 0,010 \\
Psammotermes hybostoma & 22 & 21,36 & 0,214 \\
\hline
\end{tabular}

Tableau 4 : Moyennes des espèces attaquées

\begin{tabular}{l|c}
\hline Modalités & Moyenne \\
\hline Acacia seyal & $1,00^{\mathrm{a}}$ \\
Anogeissus leiocarpa & $1,00^{\mathrm{ab}}$ \\
Mitragyna inermis & $1,00^{\mathrm{ab}}$ \\
Acacia raddiana & $1,50^{\mathrm{ab}}$ \\
Adansonia digitata & $1,50^{\mathrm{ab}}$ \\
Acacia senegal & $1,75^{\mathrm{ab}}$ \\
Grewia bicolor & $2,00^{\mathrm{ab}}$ \\
Faidherbia albida & $2,33^{\mathrm{ab}}$ \\
Guiera senegalensis & $3,00^{\mathrm{ab}}$ \\
leptadagna pirotechnica & $4,33^{\mathrm{ab}}$ \\
Calotropis procera & $5,16^{\mathrm{ab}}$ \\
Sclerocarya birrea & $5,80^{\mathrm{ab}}$ \\
Combretum glutinosum & $6,60^{\mathrm{b}}$ \\
Balanites aegyptiaca & $18,80^{\mathrm{c}}$ \\
Boscia senegalensis & $22,66^{\mathrm{d}}$ \\
\hline
\end{tabular}

L'appartenance au même groupe ( $a, b, c$ ou d) signifie qu'il n'y a pas de la différence statistiquement significative entre les effectifs observés (Test Newman-Keuls, seuil de $5 \%$ ). 


\section{Samb et al. J. Appl. Biosci. 2016 Les attaques des Termites (Isoptera) dans les parcelles de reboisement}

de la Grande Muraille Verte au Sénégal

Moyennes des attaques des espèces végétales (Tableau4) : L'analyse comparative des moyennes des espèces végétale attaquées dans les différentes parcelles révèle que ce sont les espèces Boscia senegalensis et Balanites aegyptiaca qui sont les plus attaquées avec des moyennes respectives de 22,66 et 18,80. Cela est sans doute lié à leur nombre d'individus plus élevé que celui des autres espèces. Leurs attaques se font les racines et les branches pour Boscia senegalensis. Sur le tronc pour Balanites aegyptiaca. Les attaques portées sur l'espèce Calotropis procera $(5,16)$ se font généralement sur les parties mortes de la plante.

\section{DISCUSSION}

Au total, 14 espèces de Termites attaquant les ligneux ont été récoltées dans six parcelles de reboisement situées entre Widou Thiengoly et Téssékéré. Ce nombre est inférieur à celui de Ndiaye \& Samb (2012) qui ont travaillé dans la même zone et qui avaient trouvé 16 espèces de Termites. Cette différence est sans doute liée au faite nous nous sommes intéressés uniquement aux espèces de Termites qui attaquent les ligneux alors qu'ils faisaient l'inventaire des espèces de Termites dans les parcelles. Au sud de Téssékéré, dans le Réserve de Faune du Ferlo Nord de Katané et à Danthiady (département de Matam), Gueye (2011) et Samb \& al. (2010) ont respectivement recensé 14 et 16 espèces. Dans leurs relevés figure le genre Trinervitermes qui absent dans nos relevés. Cela s'explique par le fait que c'est un genre fourrageur qui s'attaque aux herbacés. Par conséquent il ne s'attaque pas aux ligneux s'il y a la présence d'herbacés. Un plus au nord et dans la même zone climatique, à Fété Olé, Lepage (1974) a dénombré 23 espèces. La différence dans les diversités spécifiques réside essentiellement dans la plus grande diversité d'habitats échantillonnés par Lepage. En effet, cet auteur a travaillé dans 3 milieux qu'il a désignés sous les appellations de petit système dunaire, grand système dunaire et cuirasse. Notre site d'étude répond à la description du petit système dunaire de Lepage constitué de dunes de faible amplitude et d'interdune plus ou moins fermées (dépressions). C'est ce qui explique l'absence des Cubitermes uniquement signalés sur cuirasse. L'étude de la fréquence des espèces dans les différentes parcelles étudiées a montré la dominance de Psammotermes hybostoma. Dans le Ferlo septentrional cette espèce est constante dans les relevés de Lepage (1974). Par son impact, l'Homme peut entrainer la disparition de certaines espèces et favoriser le
II en est de même pour l'espèce Sclerocarya birrea $(5,80)$. La moyenne des attaques portées sur les Acacia est faible par rapport aux autres. Ces attaques ont lieu dans les parties de la plante où l'écorce est enlevée. L'attaque des Combretum se porte sur les troncs, les branches et les parties mortes. Les espèces Mitragyna inermis, Grewia bicolor et leptadagna pirotechnica sont attaquées au niveau des branches mortes. L'espèce Adansonia digitata n'est attaquée que dans ses parties sèches, même si on note des placages sur le tronc. Le Faidherbia albida est attaqué au tronc et aux branches mortes.

développement d'autres (Roy-Noël, 1978). Ainsi, du fait des activités humaines, on note un développement important de Psammotermes au niveau parcelles. Ailleurs, en Afrique, Josens (1972) qui a travaillé dans une savane en Côte d'Ivoire (Lamto-Pakobo) a rencontré, entre autres, les espèces Coptotermes intermedius, Amitermes evuncifer, Promirotermes holmgreni, Macrotermes subhyalinus, figurant dans nos relevés. Ceci montre que les espèces rencontrées dans cette zone semi-aride possèdent un potentiel écologique leur permettant de s'adapter à des conditions de milieux difficiles. Samb (2009) montre l'impact néfaste des Termites au niveau des habitations où les Termites, à la recherche de nourriture, attaquent le bois ouvré (poutre, porte, meubles...), les textiles et le papier. Au niveau des agro systèmes, ils attaquent les clôtures faites en bois, mais aussi certains arbres vivants aussi bien dans les agro systèmes que dans les zones de pâturage. Leur effet néfaste sur l'agriculture en Afrique a été signalé par des auteurs comme Brigger (1966) et Josens (1972) et sur les arbres par Han \& Ndiaye $(2006,2008)$. Ces auteurs ont montré que les Termites attaquent les cultures de Maïs et de Soja, les plants d'Eucalyptus et les arbres fruitiers. Nous avons noté une importante fréquence d'attaque par les espèces Psammotermes hybostoma, Microtermes spp. et Odontotermes spp. dans les parcelles. Ces Termites dégradent une grande masse de matière organique et transportent des quantités importantes de sol d'un horizon à un autre. D'après Mando et Brussaard (1999), les Termites contribuent à la dégradation des cannes de mil à plus de $70 \%$ dans les conditions du Sahel. L'aspect ravageur ou nuisible noté dans des milieux donnés ne doit occulter le rôle des Termites sur le sol et le fonctionnement des écosystèmes. 


\section{Samb et al. J. Appl. Biosci. 2016 Les attaques des Termites (Isoptera) dans les parcelles de reboisement}

de la Grande Muraille Verte au Sénégal

\section{REMERCIEMENTS}

Nous remercions l'Unité Mixte Internationale ESS 3189 et l'Observatoire Homme Milieux Téssékéré (Labex DRIHM) qui ont financé ce travail. Nos remerciements vont également à la population de Widou Thiengoly.

\section{BIBLIOGRAPHIE}

Adam JG : 1970. Noms vernaculaires des plantes du Sénégal. Journal d'Agric. Tropic. Et de Botanique appliquée. Tome XVII, N 7-8-9, Juillet- Sept. 1970, 112p.

Arbonnier M : 2000. Arbres, arbustes et lianes des zones d'Afrique de l'Ouest. CIRAD (Centre de Coopération International en recherche Agronomique pour le Développement) ; MNHN (Muséum National d'Histoire Naturelle) ; UICN (Union Mondiale pour la Nature), 544p.

Baumer M : 1995. Arbres et arbrisseaux nourriciers en Afrique occidentale. Enda tiers-monde, Dakar, 210-212p.

Berhaut J : 1967. Flore du Sénégal. Clairafrique, Dakar, 2 ème édition, 485p.

Bouillon A et Mathot G : 1965. Quel est ce termites africain?, E.D de l'Université Léopoldville, « zooleo » N¹, $115 \mathrm{p}$.

Brriger M: 1966. The biology and control of termite damaging field crops in Tanganyika. Bulletin of Entomological Research 56, 417-444.

Grassé PP : 1949. Ordre des Isoptères ou Termites. In Traité de Zoologie, ed. P.-P. Grassé, IX, Masson, Paris : 408-544.

Guèye $S$ : 2011. Contribution à l'étude de la faune de Termites (Isoptera) dans la Réserve de Faune du Ferlo Nord (Sénégal): cas de l'enclos d'acclimatation de Katane. Mémoire de Master II, Université Ch. A. Diop de Dakar, pp. 28.

Han SH, Ndiaye AB : 2006. Attaque des arbres fruitiers par les Termites dans la région de Kaolack (Sénégal). Bulletin de la Société Entomologique de France, 115, 123-128.

Han SH, Ndiaye AB : 2006. L'attaque des arbres fruitiers par les Termites dans la région de Thiès (Sénégal). Bulletin de la Société Entomologique de France, 111, 59-64.

Inward DJG, Vogler AP et Eggleton P: 2007. A comprehensive phylogenetic analysis of termites (Isoptera) illuminates key aspects of their volutionary biology. Molecular Phylogenetics and Evolution 44, 953-967.

Josens $G:$ 1972. Études biologique et écologique des Termites de la savane de Lamto-Pakobo (Côte d'Ivoire). Thèse de doctorat, 166-198.
Lepage M : 1974. Les Termites d'une savane sahélienne (Ferlo Septentrional, Sénégal) : peuplement, population, consommation, rôle dans l'écosystème. Thèse d'État, Université de Dijon, $344 \mathrm{p}$.

Mando A et Brussard L : 1999. Contribution of termites to the breakdown of straw under Sahelian conditions. Biology and Fertility of soils, 29: 332334.

Michel $P$, Naegele A et Toupet C : 1969. Contribution à l'étude du Sénégal septentrional. Bulletin de l'IF AN, 16 (3), 756-839.

Ndiaye AB et Samb T: 2012. Les termites (Isoptera) dans les parcelles de reboisement de la grande muraille verte entre WidouThiengoly et Tessekere (Sénégal). Les cahiers de l'Observatoire International "Homme-Milieu » Tessekere, $N^{\circ}$ 1, 63-73.

Okullo P et Moe SR : 2012. Termite activity, not grazing, is the main determinant of spatial variation in savanna herbaceous vegetation. Journal of Ecology, 100, 232-241.

Roy-Noël J: 1978. Influence de l'Homme sur le peuplement en Termites dans la presqu'ile du Cap-Vert (Sénégal occidental). Memorabilia Zoologica, 20 : 157-172.

Samb T, Ba CHT et Ndiaye AB : 2014. Étude de l'effet des de cannes du petit mil et de la fumure d'ovins et de caprins sur la macrofaune du sol en zone sahélienne (Matam, Sénégal), Bulletin de l'IFAN Ch. A. Diop, sér. A, 2014, 53 (2) : 101115

Samb T, Diarra K et Ndiaye AB: 2010. Biodiversity of Termites in Relation to Human Activity: Impact on the Environment in Matam (Senegal), Research Journal of Pharmaceutical, Biological and Chemical Sciences, vol. 2(1), p. 313-323

Samb T: Étude des Termites (Isoptera) dans les habitations, les agro systèmes et les pâturages dans une savane sahélienne à Matam (Sénégal). Mémoire de DEA, Université Ch. A. Diop de Dakar, pp. 51.

Silvestri F : 1914-15. Contribuzione alla conoscenza dei Termitidi e Termitophili dell'Africa occidentale. I. Termitidi. Bolletino del Laboratorio di Zoologia 
Samb et al. J. Appl. Biosci. 2016 Les attaques des Termites (Isoptera) dans les parcelles de reboisement de la Grande Muraille Verte au Sénégal

Generale e Agraria della R. Scuola Superore d'Agricoltura, Portici, 9, 16146.

Sjöstedt Y: 1925. Revision der Termiten Afrikas. 3. Monographie. Kungl Svenska Vetenska Akademiens Handlingar, 3 (1), 1-435.
Wood TG: 1975. The effects of clearing and grazing on the Termite Fauna (Isoptera) of Tropical Savannas and Woodlands Dans Progress in soil zoology, Publising House of Czechoslovak Academy of sciences, Prague (Ed), 409-418. 